\title{
Review of: "Impact of crowded environments on binding between protein and single-stranded DNA"
}

\author{
Gregory Poon ${ }^{1}$, Tyler Vernon ${ }^{1}$ \\ 1 Georgia State University
}

Potential competing interests: The author(s) declared that no potential competing interests exist.

\section{Background}

Cells retain large amounts of macromolecules within their plasma membranes, up to $400 \mathrm{~g} / \mathrm{L}$ depending on their physiologic state. ${ }^{[1]}$ Mutual exclusion is a fundamental property of polymers in solution. ${ }^{[2][3]}$ Classically, this excluded volume effect is treated as hard-core repulsion: an entropic depletion force that drives folding and interactions towards more compact states. ${ }^{[4][5]}$ More recent studies show that depletion forces are supplemented by lower-potential "soft" interactions which may even be attractive. ${ }^{[6][7][8][9][10]}$ While the impact of crowders on protein stability and dynamics in solution has been recognized since the early works of Ogston ${ }^{[11]}$ and Laurent ${ }^{[12]}$, their theoretical treatment continues to be debated. [13][14][15][16][17][18] Experimental measurements remain a mainstay of studying biological interactions in a crowded environment.

In their study, Köhn et al. characterize the impact of macromolecular crowding on the binding of singlestranded DNA, specifically oligothymidines ( $d T n, n=5$ to 7 nucleotides) by cold shock protein B from Bacillus subtilis (BsCspB, 67 residues), using polyethylene glycol (PEG1 and PEG8, 1k and 8k), ethylene glycol (EG), dextran (Dex20, 20k), and glucose as cosolutes. Monomer-polymer pairs enable a comparison of both chemical composition and size. On the one hand, PEG and EG act more like an organic solvent, which displays hydrophobic tendencies. On the other hand, Dex and glucose are more polar. The authors hypothesize that weak chemical interactions dominate excluded volume effects in crowding protein/DNA binding.

\section{Technical review}

BsCspB structure is conserved under crowding. High concentrations of any cosolute always raises a concern of specific interactions that would obscure the intended interpretation of the data. To assess this possibility, the authors used heteronuclear NMR spectroscopy to probe the equilibrium structures of free BsCspB and dTn-bound complexes. An extensive series of $1 \mathrm{D}\left({ }^{1} \mathrm{H}\right.$ and $\left.{ }^{31} \mathrm{P}\right)$ and $2 \mathrm{D}$ $\left({ }^{1} \mathrm{H}_{-}{ }^{15} \mathrm{~N} \mathrm{HSQC}\right.$ and ${ }^{1} \mathrm{H}_{-}{ }^{13} \mathrm{C}$ HSQC) experiments showed substantially similar chemical shift perturbations (CSP) or intensity in both the protein and DNA upon binding whether in dilute solution or up to $300 \mathrm{~g} / \mathrm{L}$ of crowding agent. While the CSP data is presented as overlays of the free and bound states under identical 
conditions in the main text (e.g., Figure 1), overlays of free or variously bound spectra under dilute and crowded conditions may also be informative. Regardless, NMR evidence under neutral buffered conditions suggests that crowding agents do not drastically alter the average structure of BsCspB upon binding at the residue level.

\section{Crowding decreases BsCspB/ssDNA affinity and kinetics in a crowder-dependent manner.} The authors used steady-state fluorescence spectroscopy (based on intrinsic tryptophan fluorescence quench upon binding) to determine $B s \mathrm{CspB} / \mathrm{dTn}$ binding affinity at equilibrium, identifying differences between solutions treated with the different treatments. The presence of PEG or EG significantly reduced dTn binding affinity for BsCspB. Limited data for PEG1 and PEG8 in dT7 binding showed little incremental effect by the larger crowder. In contrast, equivalent \% w/v glucose was much less perturbative than EG, and crowding by Dex20 produced the opposite effect, enhancing dTn affinity with increasing concentration. While the MW of glucose is about three times that of EG, it is difficult to discern whether the decrease in affinity by EG and glucose (each measured at a single \%w/v, representing two different activities) represented cosolute interactions or colligative hydration effects. The divergent effects of glucose and Dex20 on binding affinity demonstrate the impact of cosolute size on binding, which oppose and overcome the negative contributions by the monomer.

To tease out the subtle binding affinity differences, the authors used stopped-flow techniques to quantify the kinetics of $B s \mathrm{CspB} / \mathrm{dTn}$ binding. Remarkably, the association rate constant $\left(\mathrm{k}_{\mathrm{on}}\right)$ of the complex decreases linearly with the \%w/v of the crowding agent essentially independently of cosolute identity and size (Table 3G). In contrast, the dissociation rate constant $k_{\text {off }}$ differed widely depending on the cosolute (Table 3H). While the polymers (PEGI and Dex20) showed relatively little effect on $\mathrm{k}_{\text {off }}$ out to $300 \mathrm{~g} / \mathrm{L}$, their monomers produced qualitatively opposite differences: EG increased $\mathrm{k}_{\text {off }}$ monotonically with concentration, whereas glucose sharply decreased $k_{\text {off }}$ at $100 \mathrm{~g} / \mathrm{L}$ before showing no additional effect (Table $3 \mathrm{H})$. The non-monotonic effect of glucose seems to imply complex effects of glucose on the ratelimiting step in the dissociation of the BsCspB/dTn complex. Taken together, the authors conclude that chemical interactions dominate in crowding. Interestingly, the presented kinetic parameters generally underestimate the equilibrium dissociation constants $\left(K_{D}=k_{o f f} / k_{o n}\right)$ by a factor of two or more, suggesting the potential of steps in BsCspB/dTn binding that are not fully captured by the 1:1 model used to fit the equilibrium data.

Cosolute effects on solvent exchange by BsCspB. Given the apparent dissociation kinetics, the authors returned to NMR to interrogate (unbound) BsCspB by amide hydrogen exchange (Figure 4). At equivalent \%w/v (240 g/mL), PEG1 and Dex20 were generally less perturbative on the solvent exchange rate constant $\left(k_{e x}\right)$ than their monomers, whose effects were most pronounced at residues with rapid solvent exchange under dilute conditions. Noting a rough correspondence in the trends in $\mathrm{k}_{\mathrm{ex}}$ and the dissociation rate constant, the authors proposed that cosolute effects in dTn binding are mediated through the "hydration shell" around BsCspB.

Amide hydrogen exchange is most directly interpreted in terms local solvent accessibility and 
reactivity of proton exchange. In previous work, the authors have correlated solvent accessibility with the conformational stability of free BsCspB. ${ }^{[19]}$ In the present study, the authors acknowledge that the solvent exchange profiles only partially explain the dissociation kinetics data. Namely, there was no obvious difference in the exchange profiles for EG and glucose that would track their quantitative effects on dTn binding affinity. Nor was there any pattern in the corresponding profiles for PEG1 and Dex20 to explain the qualitatively opposite effects of PEG and Dex20 on binding affinity. Uncertainty may be partly due to a lack of data on the dTn-bound complex itself, or at least unbound dTn. Another confounding factor may be uncontrolled cosolute effects on conformation and binding on the one hand, and the intrinsic reactivity of proton exchange with solvent on the other, for which resolution requires measurement over a range of $\mathrm{pH}$.

\section{Perspective}

To place the crowding effects from this study in perspective, it is instructive to consider the relative sizes (e.g., radii of gyration, $\mathrm{Rg}$ ) of the macromolecules involved. Using their crystal structures, the $\mathrm{Rg}$ of $B s \mathrm{CspB}$ (PDB: 1CSP) and a BsCspB/dT6 complex (2ES2) may be computed to be 1.1 and $1.3 \mathrm{~nm}$, respectively, which would somewhat underestimate their true values as fluctuating folded states in solution. For free dTn, we may estimate an $\mathrm{Rg}$ up to $0.7 \mathrm{~nm}$ for dT7 based on SAXS measurements of poly-dT. ${ }^{[20]}$ Solution dimensions of PEG and Dex, which are commercially important excipients, are extensively reported in the literature:[21][22][23] $\mathrm{Rg} \sim 1.0 \mathrm{~nm}$ for PEG1, $\sim 2.0 \mathrm{~nm}$ for PEG8, and $\sim 2.2 \mathrm{~nm}$ for Dex20. These crowding agents are therefore the same order in size as BsCspB and dTn. One wonders how the apparent balance of "soft" and "hard" effects of crowding might depend on crowder size and whether excluded-volume effects might increasingly contribute if much larger crowders were studied.

Overall, the authors make a case for soft interactions on ligand binding of proteins, following a "bottom-up" approach that prioritizes defined chemical characteristics. As a result, the authors gained insight into physicochemical properties driving crowding of $B s \mathrm{CspB} / \mathrm{dTn}$ binding, justifying their choice of synthetic crowders over naturally derived crowders such as reconstituted cell lysate. That said, the use of one model system and two pairs of cosolutes necessarily limits the generalizability of the results from a biological relevance perspective.

\section{Paper in context}

Research into the binding properties of transcription factors under native-like conditions bears implications for our understanding of gene expression, as the authors stress in their paper. Biophysical data on crowded protein/DNA interactions remains limited relative to protein folding and interactions, DNA stability, and diffusion. [24][25][26][27][28][29][30] Interest has recently arisen on how crowding modifies target site search via DNA-mediated diffusion. ${ }^{[31][32][33]}$ Nevertheless, there has been limited progress in relating the stability and interactions of nucleoprotein complexes in dilute solution, on which a large body of literature exists, to their behavior under crowded conditions. The welcome study by Köhn et al. shows that crowding 
with PEG and Dex20 can significantly impact, in an identity-dependent manner, single-stranded DNA binding by BsCspB, without exerting major structural changes on the protein.

In the literature, attribution to soft interactions in macromolecular crowding remains somewhat secondary to excluded-volume effects. Binding studies that report similar observations as Köhn et al., namely a lack of major structural change upon crowding and the volume of proteins affecting binding, frequently favor hard interactions as the primary contributor. It may be the case that binding (wherein at least the receptor or ligand is well folded) is more sensitive to soft interactions, whereas conformational transitions involving highly unfolded structures are more sensitive to excluded-volume effects. The relative effectiveness of low-MW cosolutes to exert osmotic pressure is generally well recognized. On the flip side, while all cosolutes make exclusion contributions in solution ${ }^{[34]}$, excluded volumes scale with the effective radius cubed. Low-MW cosolutes, therefore, do not yield the crowding effects of polymers at experimentally relevant $\% \mathrm{w} / \mathrm{v}$. Parallel evaluation of constituent monomers of polymeric crowders thus represents a wellreasoned control for helping resolve the two classes of contributions.

In closing, we regret the omission of numerous colleagues who contribute to our understanding of macromolecular crowding in our attempt for concision.

\section{References}

1. ^M. T. Record, Jr., E. S. Courtenay, D. S. Cayley, H. J. Guttman. (1998). Responses of E. coli to osmotic stress: large changes in amounts of cytoplasmic solutes and water. Trends Biochem Sci. 23(4):143-148. Retrieved from https://www.ncbi.nlm.nih.gov/pubmed/9584618. PubMed PMID: 9584618.

2. ^Paul J. Flory. (1953). Principles of polymer chemistry. ISBN 08014013489780801401343.

3. Robert L. Scott. (1949). The Thermodynamics of High Polymer Solutions. V. Phase Equilibria in the Ternary System: Polymer 1-Polymer 2-Solvent. The Journal of Chemical Physics. 17(3):279-284. doi:10.1063/1.1747239.

4. `Sho Asakura, Fumio Oosawa. (1958). Interaction between particles suspended in solutions of macromolecules. J Polym Sci. 33(126):183-192. doi:10.1002/pol.1958.1203312618.

5. AAllen P. Minton. (1981). Excluded volume as a determinant of macromolecular structure and reactivity. Biopolymers. 20(10):2093-2120. doi:10.1002/bip.1981.360201006.

6. ^Y. Wang, M. Sarkar, A. E. Smith, A. S. Krois, G. J. Pielak. (2012). Macromolecular crowding and protein stability. J Am Chem Soc. 134(40):16614-16618. doi:10.1021/ja305300m. PubMed PMID: 22954326.

7. ^A. P. Minton. (2013). Quantitative assessment of the relative contributions of steric repulsion and chemical interactions to macromolecular crowding. Biopolymers. 99(4):239-244. doi:10.1002/bip.22163. PubMed PMID: 23348671; PubMed Central PMCID: PMCPMC3557810.

8. `Liel Sapir, Daniel Harries. (2015). Is the depletion force entropic? Molecular crowding beyond steric interactions. Current Opinion in Colloid \& Interface Science. 20(1):3-10.

doi:10.1016/j.cocis.2014.12.003. 
9. ^A. C. Miklos, M. Sarkar, Y. Wang, G. J. Pielak. (2011). Protein crowding tunes protein stability. J Am Chem Soc. 133(18):7116-7120. doi:10.1021/ja200067p. PubMed PMID: 21506571.

10. ^M. Sarkar, C. Li, G. J. Pielak. (2013). Soft interactions and crowding. Biophys Rev. 5(2):187-194. doi:10.1007/s12551-013-0104-4. PubMed PMID: 28510157; PubMed Central PMCID: PMCPMC5418433.

11. ^A. G. Ogston. (1958). The spaces in a uniform random suspension of fibres. Transactions of the Faraday Society. 54(0):1754-1757. doi:10.1039/TF9585401754.

12. `T. C. Laurent. (1964). The interaction between polysaccharides and other macromolecules. 9. The exclusion of molecules from hyaluronic acid gels and solutions. Biochem J. 93(1):106-112. Retrieved from https://Www.ncbi.nlm.nih.gov/pubmed/4158275. PubMed PMID: 4158275; PubMed Central PMCID: PMCPMC1206188.

13. A. Politou, P. A. Temussi. (2015). Revisiting a dogma: the effect of volume exclusion in molecular crowding. Curr Opin Struct Biol. 30:1-6. doi:10.1016/j.sbi.2014.10.005. PubMed PMID: 25464122.

14. `Y. Phillip, E. Sherman, G. Haran, G. Schreiber. (2009). Common crowding agents have only a small effect on protein-protein interactions. Biophys J. 97(3):875-885. doi:10.1016/j.bpj.2009.05.026. PubMed PMID: 19651046; PubMed Central PMCID: PMCPMC2718159.

15. `Damien Hall, Allen P. Minton. (2003). Macromolecular crowding: qualitative and semiquantitative successes, quantitative challenges. Biochim Biophys Acta Protein Proteomics. 1649(2):127-139. doi:10.1016/s1570-9639(03)00167-5.

16. `V. A. Parsegian, R. P. Rand, D. C. Rau. (2000). Osmotic stress, crowding, preferential hydration, and binding: A comparison of perspectives. Proc Natl Acad Sci U S A. 97(8):3987-3992. doi:10.1073/pnas.97.8.3987.

17. ^ L. A. Benton, A. E. Smith, G. B. Young, G. J. Pielak. (2012). Unexpected effects of macromolecular crowding on protein stability. Biochemistry. 51(49):9773-9775. doi:10.1021/bi300909q. PubMed PMID: 23167542.

18. ^P. R. Davis-Searles, A. J. Saunders, D. A. Erie, D. J. Winzor, G. J. Pielak. (2001). Interpreting the effects of small uncharged solutes on protein-folding equilibria. Annu Rev Biophys Biomol Struct. 30:271-306. doi:10.1146/annurev.biophys.30.1.271. PubMed PMID: 11340061.

19. ^B. Kohn, M. Kovermann. (2019). Macromolecular Crowding Tunes Protein Stability by Manipulating Solvent Accessibility. Chembiochem. 20(6):759-763. doi:10.1002/cbic.201800679. PubMed PMID: WOS:000461832400004.

20. ^A. Y. Sim, J. Lipfert, D. Herschlag, S. Doniach. (2012). Salt dependence of the radius of gyration and flexibility of single-stranded DNA in solution probed by small-angle $x$-ray scattering. Phys Rev E Stat Nonlin Soft Matter Phys. 86(2 Pt 1):021901. doi:10.1103/PhysRevE.86.021901. PubMed PMID: 23005779.

21. `J. K. Armstrong, R. B. Wenby, H. J. Meiselman, T. C. Fisher. (2004). The hydrodynamic radii of macromolecules and their effect on red blood cell aggregation. Biophys J. 87(6):4259-4270. doi:10.1529/biophysj.104.047746. PubMed PMID: 15361408; PubMed Central PMCID: PMCPMC1304934. 
22. ${ }^{\wedge}$ R. Bhat, S. N. Timasheff. (1992). Steric exclusion is the principal source of the preferential hydration of proteins in the presence of polyethylene glycols. Protein Sci. 1(9):1133-1143.

doi:10.1002/pro.5560010907. PubMed PMID: 1304392; PubMed Central PMCID: PMCPMC2142180.

23. `K. L. Linegar, A. E. Adeniran, A. F. Kostko, M. A. Anisimov. (2010). Hydrodynamic radius of polyethylene glycol in solution obtained by dynamic light scattering. Colloid Journal. 72(2):279-281. doi:10.1134/s1061933x10020195.

24. `A. Christiansen, Q. Wang, M. S. Cheung, P. Wittung-Stafshede. (2013). Effects of macromolecular crowding agents on protein folding in vitro and in silico. Biophys Rev. 5(2):137-145. doi:10.1007/s12551-013-0108-0. PubMed PMID: 28510156; PubMed Central PMCID: PMCPMC5418436.

25. ^B. van den Berg, R. J. Ellis, C. M. Dobson. (1999). Effects of macromolecular crowding on protein folding and aggregation. EMBO J. 18(24):6927-6933. doi:10.1093/emboj/18.24.6927. PubMed PMID: 10601015; PubMed Central PMCID: PMCPMC1171756.

26. `D. Miyoshi, N. Sugimoto. (2008). Molecular crowding effects on structure and stability of DNA. Biochimie. 90(7):1040-1051. doi:10.1016/j.biochi.2008.02.009. PubMed PMID: 18331845.

27. `J. A. Dix, A. S. Verkman. (2008). Crowding effects on diffusion in solutions and cells. Annu Rev Biophys. 37:247-263. doi:10.1146/annurev.biophys.37.032807.125824. PubMed PMID: 18573081.

28. `S. I. Nakano, N. Sugimoto. (2016). Model studies of the effects of intracellular crowding on nucleic acid interactions. Mol Biosyst. 13(1):32-41. doi:10.1039/c6mb00654j. PubMed PMID: 27819369.

29. `S. Nakano, D. Miyoshi, N. Sugimoto. (2014). Effects of molecular crowding on the structures, interactions, and functions of nucleic acids. Chem Rev. 114(5):2733-2758. doi:10.1021/cr400113m. PubMed PMID: 24364729.

30. ^I. M. Kuznetsova, K. K. Turoverov, V. N. Uversky. (2014). What Macromolecular Crowding Can Do to a Protein. International Journal of Molecular Sciences. 15(12):23090-23140. doi:10.3390/ijms151223090. PubMed PMID: WOS:000346797400091.

31. ^A. Mahmutovic, O. G. Berg, J. Elf. (2015). What matters for lac repressor search in vivo--sliding, hopping, intersegment transfer, crowding on DNA or recognition? Nucleic Acids Res. 43(7):3454-3464. doi:10.1093/nar/gkv207. PubMed PMID: 25779051; PubMed Central PMCID: PMCPMC4402528.

32. ^P. Dey, A. Bhattacherjee. (2018). Role of Macromolecular Crowding on the Intracellular Diffusion of DNA Binding Proteins. Sci Rep. 8(1):844. doi:10.1038/s41598-017-18933-3. PubMed PMID: 29339733; PubMed Central PMCID: PMCPMC5770392.

33. ^A. A. Shvets, A. B. Kolomeisky. (2016). Crowding on DNA in Protein Search for Targets. J Phys Chem Lett. 7(13):2502-2506. doi:10.1021/acs.jpclett.6b00905. PubMed PMID: 27314905.

34. ^T. V. Chalikian. (2016). Excluded volume contribution to cosolvent-mediated modulation of macromolecular folding and binding reactions. Biophys Chem. 209:1-8. doi:10.1016/j.bpc.2015.11.001. PubMed PMID: 26569082. 
